\title{
Acupuncture Compared with Intramuscular Injection of Neostigmine for Postpartum Urinary Retention: A Systematic Review and Meta-Analysis of Randomized Controlled Trials
}

\author{
Xiao-Mei Wang, ${ }^{1}$ Jing Gong, ${ }^{2}$ Si-Cong $\mathrm{Li}^{3}{ }^{3}$ and Mei Han $\mathbb{D}^{3}$ \\ ${ }^{1}$ Beijing Obstetrics and Gynecology Hospital, Capital Medical University, Beijing, China \\ ${ }^{2}$ Beijing Anzhen Hospital, Capital Medical University, Beijing, China \\ ${ }^{3}$ Beijing University of Chinese Medicine, Beijing, China \\ Correspondence should be addressed to Mei Han; hanmeizoujin@163.com
}

Received 24 December 2017; Accepted 12 April 2018; Published 14 May 2018

Academic Editor: Caigan Du

Copyright (C) 2018 Xiao-Mei Wang et al. This is an open access article distributed under the Creative Commons Attribution License, which permits unrestricted use, distribution, and reproduction in any medium, provided the original work is properly cited.

\begin{abstract}
Objective. To compare the effectiveness and safety of acupuncture versus intramuscular injection of neostigmine. Methods. Databases including CNKI, VIP, WanFang, SinoMed, PubMed, Cochrane Library, and clinicaltrials.gov database were retrieved for relevant literature, with the retrieval deadline being November 2017. Two reviewers independently screened, selected, and extracted data and validated the results. The methodological quality was evaluated with the "Risk of Bias" tool, and the meta-analysis was performed by using the RevMan 5.3.5 software. Results. Totally 953 patients with postpartum urinary retention from 15 randomized controlled trials entered the meta-analysis. 12 articles compared the clinical cure rate of acupuncture alone versus intramuscular injection of neostigmine and found the cure rate in the acupuncture group was 2 times that in the neostigmine group (RR, 1.91; 95\% CI: 1.66-2.19). 15 articles compared the clinical effectiveness rate of acupuncture alone with that of intramuscular injection of neostigmine and found the clinical effectiveness rate was $28 \%$ higher in the acupuncture group than in the neostigmine group (RR: 1.28; 95\% CI: 1.16-1.42). No adverse event was reported in the acupuncture group. Conclusion. Acupuncture alone is more effective in treating postpartum urinary retention than intramuscular injection of neostigmine, with good safety profile. Therefore, it is a feasible and valuable technique in clinical settings.
\end{abstract}

\section{Introduction}

Postpartum urine retention (PUR) refers to the inability to pass urine for 6 to 8 hours after delivery. Clinically it is manifested as weak dribble of urine or completely blocked urinary tract, accompanied by abdominal distention and pain (symptomatic PUR) [1]; or patients are able to void spontaneously but have a postvoid residual bladder volume (PVRV) of $\geq 150 \mathrm{~mL}$ (symptomatic PUR) [2]. Reported prevalence for overt symptomatic PUR ranges from $0.3 \%$ to $4.7 \%[3,4]$. For asymptomatic PUR, prevalence of even up to $45 \%$ has been reported [5]. It can increase postpartum hemorrhage and is not conducive to milk secretion [6].

Postpartum urinary retention is initially treated with routine methods for inducing urination induction. When these routine methods fail, medications (neostigmine, phenoxybenzamine, and enema), acupuncture, and/or catheterization may be applied. Although catheterization is the most effective way to relieve urinary retention, it can increase the risk of urinary tract infection [7]. Intramuscular injection of neostigmine is only effective in $70 \%$ of patients, and it is associated with many side effects such as bradycardia, bronchoconstriction, increased secretions, nausea, and vomiting [8]. Therefore, more safe and effective treatments need to be explored and applied.

Acupuncture, as a nondrug therapy, has been widely used in the treatment of various diseases both in China and abroad, especially for pain symptoms and nerve dysfunction. Urination of the bladder is a reflex activity of nerves. Modern medical studies have proved that acupoints are quite consistent with the corresponding viscera in the ganglions. For urinary retention patients with intact bladder 
nerves, acupuncture of acupoints including pangguangshu, zhongji, ciliao, and shenshu can stimulate the conduction of parasympathetic nerve impulses via nerve reflex, resulting in contraction of bladder detrusor, increase of intravesical pressure, and relaxation of internal sphincter; by doing so, it can regulate the urination function of the bladder [9]. As a safe, effective, and efficient therapy, acupuncture has become increasingly popular among patients. Meanwhile, a large number of studies have investigated the role of acupuncture in treating postpartum urinary retention. In our current analysis, we compared the effectiveness and safety of acupuncture versus intramuscular injection of neostigmine, a commonly used drug, in the treatment of urinary retention.

\section{Methods}

2.1. Inclusion/Exclusion Criteria. The subjects were mothers with clinically confirmed postpartum urinary retention; the study type was a randomized controlled trial; the interventions included acupuncture (hand acupuncture or electroacupuncture) alone, with unrestricted acupoints or maneuvers; and the control group was treated with intramuscular injection of neostigmine.

2.2. Literature Review. Published literature was retrieved in databases including China National Knowledge Infrastructure (CNKI), WanFang Data, SinoMed, CQVIP, PubMed, and Cochrane Library. In the clinicaltrials.gov website we also retrieved the completed but unpublished studies and tracked the results of these studies. Only Chinese and English articles were retrieved, and the deadline was October 2017. The Chinese retrieval words included "postpartum urinary retention", "acupuncture”, "electroacupuncture", "scalp acupuncture", "three-edged needle", "plum-blossom needle", "filiform needle", and "randomization", and the English retrieval words included "urinary retention", "acupuncture", "acusector", "needle", "needling", "randomized controlled trial", "clinical trial", "RCT", "random", and "randomization". Based on the characteristics of different databases, retrieval strategies including both subject words + free words and key words + full text were applied.

2.3. Literature Screening. The initially identified articles were imported into NoteExpress. Initial screening was performed based on inclusion/exclusion criteria and after reading literature titles and abstracts. Full texts were acquired and read for eligible articles or articles that might meet the inclusion criteria to decide whether they would enter the final analysis.

2.4. Data Extraction. A data extraction table was designed to collect the data to be analyzed, which included the following seven aspects: (a) basic information of the enrolled studies; (b) research methods and their possible biases; (c) features of the subjects; (d) interventions; (e) outcome measures; (f) research findings; and (g) other required information. Two reviewers independently extracted these data and validated the findings.

2.5. Quality Evaluation. The risk of bias was assessed according to the Cochrane Handbook for Systematic Reviews of
Interventions [10], which covered the random sequence generation, allocation concealment, blinding, data integrity, selective reporting of positive and/or negative findings, and other sources of bias. Among them, the "other sources of bias" included the following: (a) were there clear inclusion/exclusion criteria; (b) were the baseline data comparable; and (c) was there any conflict of interest. For selective outcome reporting, since all the included studies did not register their research protocols, the risk of bias was defined as low if the outcome measures included postvoiding residual (PVR) and as high if no PVR was reported. The risk of bias was assessed and validated independently by two reviewers; the results were cross-referenced and any disagreements were dissolved by discussion or consultation of a third evaluator with rich experiences.

2.6. Outcome Measures. Primary endpoints are cure rate and PVR; secondary endpoints are overall effectiveness rate; and safety outcome was adverse events.

2.7. Data Analysis. The quantitative analysis was carried out by using the Cochrane Collaboration software RevMan5.3.5 for meta-analysis. The relative risk (RR) was selected as the statistic for dichotomous data; the continuous variables are described using mean difference (MD) and 95\% confidence interval (CI). During the heterogeneity test, chi-square test was performed firstly; based on its finding, estimates of heterogeneity $\left(I^{2}\right)$ were applied. Fixed-effect model was used when $I^{2}$ was $\leq 50 \%$ and the $P$ value was $\geq 0.10$; when $I^{2}$ was $>50 \%$ or the $P$ value was $<0.10$, random-effect model was applied. If heterogeneity is high, the source for heterogeneity was explored; subgroup analysis or sensitivity analysis was performed to investigate the stability of meta-analysis.

2.8. Measurement of Publication Bias. When a sufficient number of articles (more than 10) were included under the same endpoint addressing the same question (more than 10), a funnel plot was used to measure the publication bias.

\section{Results}

3.1. Literature Searching and Screening Flowchart. Totally 667 articles were retrieved based on our retrieval strategies. After removing duplication and screening the titles and abstracts, we obtained 112 full-text articles. Fifteen articles entered the final analysis according to the exclusion criteria (Figure 1).

3.2. Characteristics of Included Literature. A total of 15 randomized controlled trials (RCTs, involving 953 patients) [1125] on the treatment of postpartum urinary retention by acupuncture alone or intramuscular injection of neostigmine were included in our analysis. The main acupoints used in these studies included sanyinjiao, zhongji, zusanli, guanyuan, yinlingquan, sanjiao, shuidao, and zhiyin. The specific features of these studies are summarized in Table 1.

3.3. Quality Evaluation of the Articles. Among these 15 studies, patients were randomized by using random number table in three studies $[16,20,24]$ and by using the SPSS 


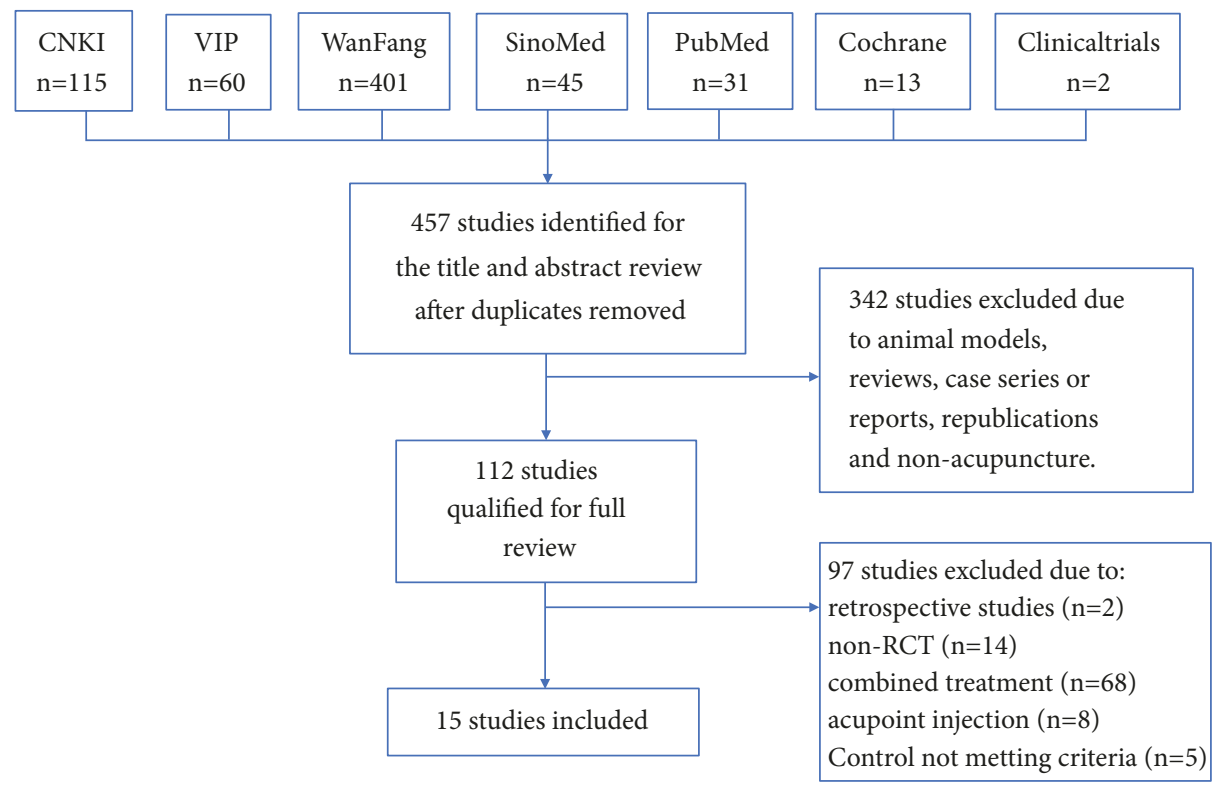

FIGURE 1: The literature searching and screening flowchart.

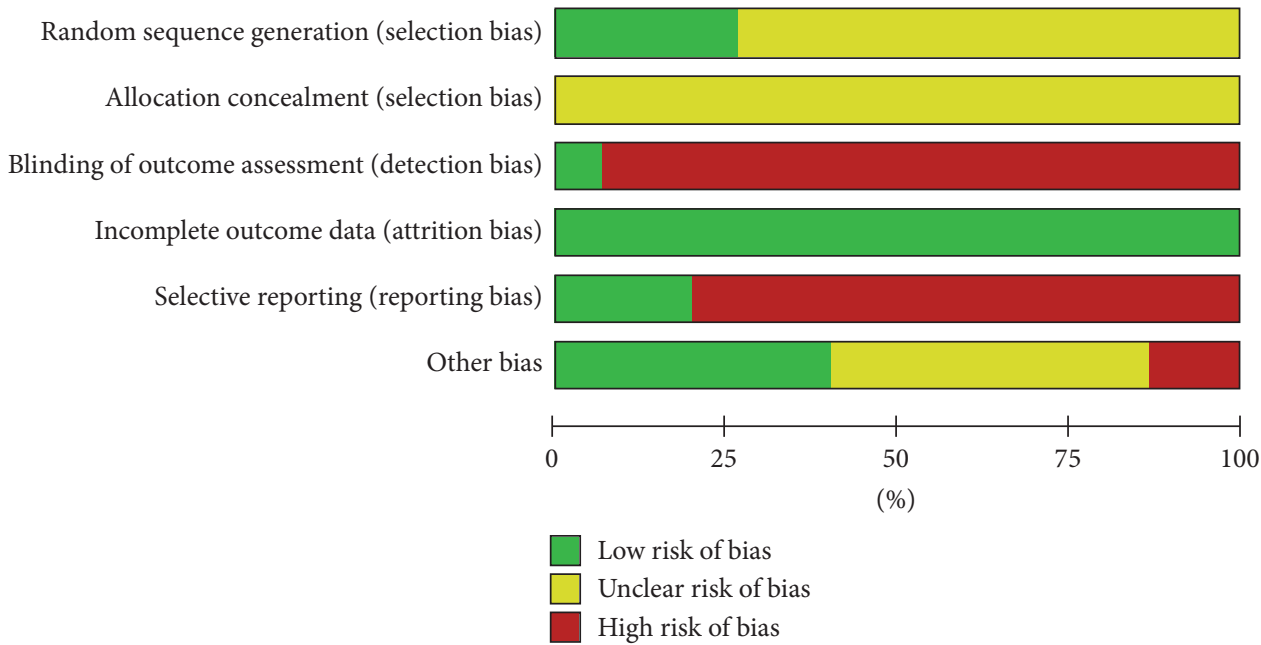

FIGURE 2: Evaluation of the risk biases of the included studies.

software in one study [15]; the remaining 11 studies only mentioned "random" or "randomization," without describing the specific randomization methods. No literature described allocation concealment. One literature described the blinding of outcome measurers and data analysts [15]. In all literature, the number of patients in all the randomized groups was consistent with the number of subjects in the statistical analysis. Only two articles reported both clinical effectiveness rate and PVR, without selective outcome reporting $[15,21]$. The remaining 13 articles only reported the CRR and failed to adequately report RCTs according to the Consolidated Standards of Reporting Trials (CONSORT) statement. The quality of the literature included in our analysis was average. The details of the evaluation are shown in Figure 2.

\subsection{Results of Meta-Analysis}

3.4.1. Cure Rate. 12 of these 15 studies compared the cure rate. While the definition of "cure" differed among these studies, it mainly includes the time of the ability to urinate after treatment $(15 \mathrm{~min}$ to $3 \mathrm{~h}$ was described in 7 trials and insufficient information in the other 4 trials), absence of distending pain in the lower abdomen, and absence of the urgent urination, a PVR of $<50 \mathrm{ml}$, and nonrecurrence. Test for between-studies heterogeneity of 12 studies yielded an $I^{2}$ of $34 \%$. The pooled cure rate was $71.91 \%$ in the acupuncture group and $38.58 \%$ in the neostigmine group; the RR (and 95\% CI) was 1.91 $(1.66,2.19)$ when these two groups were compared in the fixed-effect model, suggesting that acupuncture had a higher 


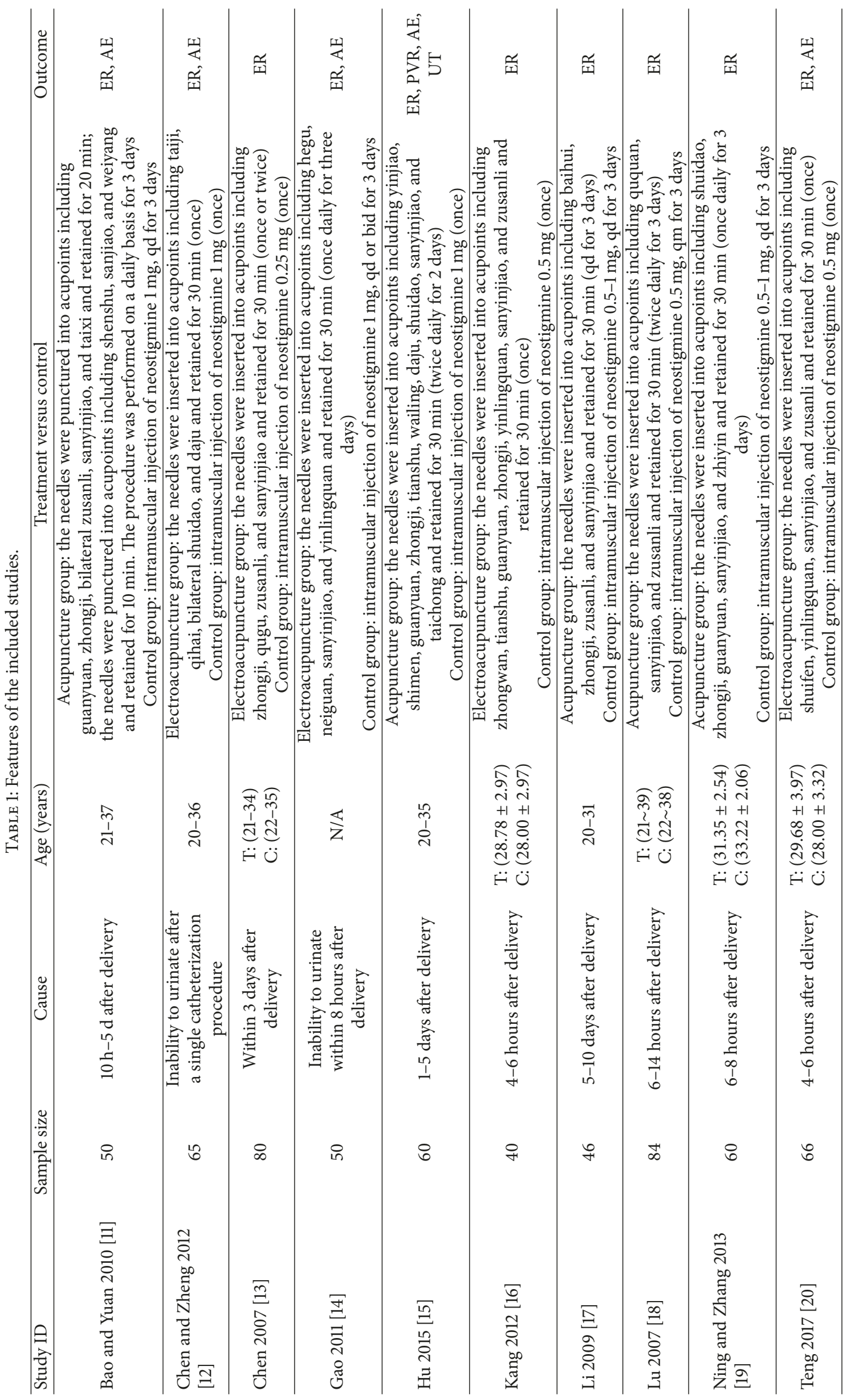




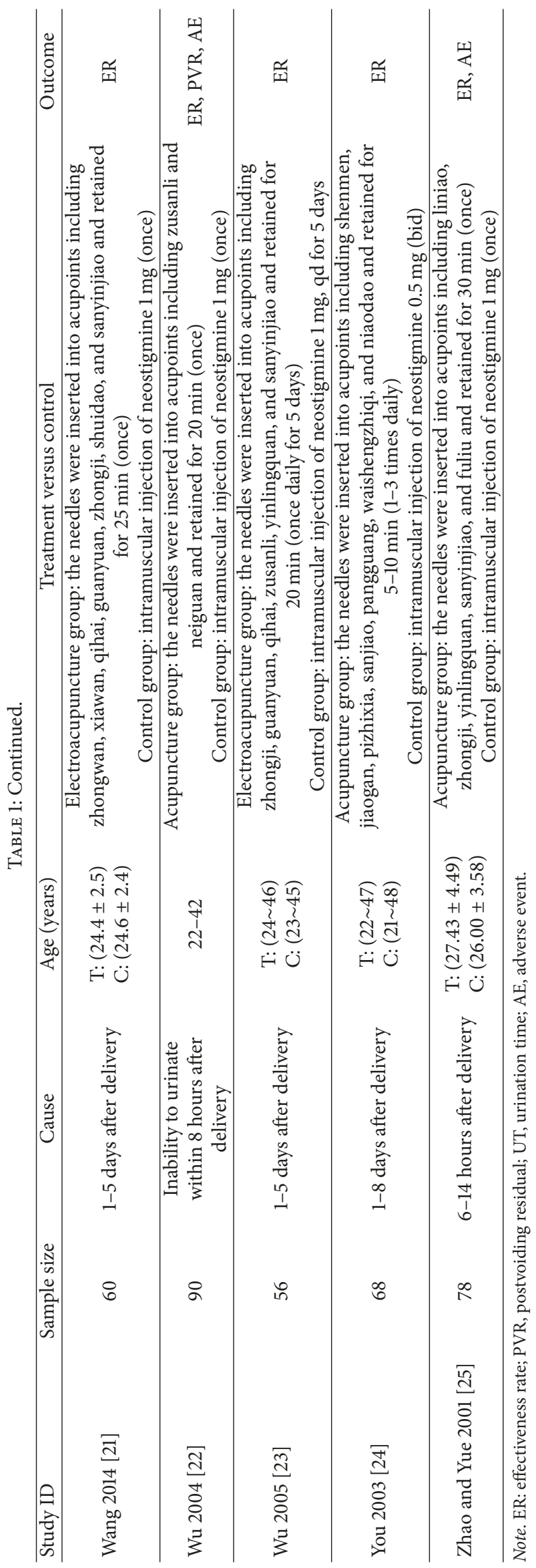




\begin{tabular}{|c|c|c|c|c|c|c|c|c|c|}
\hline \multirow{3}{*}{$\begin{array}{l}\text { Study or Subgroup } \\
\text { Bao FF } 2010\end{array}$} & \multicolumn{2}{|c|}{ Experimental } & \multicolumn{2}{|c|}{ Control } & \multirow{3}{*}{$\begin{array}{c}\text { Weight } \\
4.6 \%\end{array}$} & \multirow{2}{*}{$\begin{array}{c}\text { Risk Ratio } \\
\text { M-H, Fixed, 95\% CI }\end{array}$} & \multirow{2}{*}{\multicolumn{3}{|c|}{$\begin{array}{c}\text { Risk Ratio } \\
\text { M-H, Fixed, 95\% CI }\end{array}$}} \\
\hline & \multirow{2}{*}{$\begin{array}{c}\text { Events } \\
19\end{array}$} & \multirow{2}{*}{$\frac{\text { Total }}{26}$} & \multirow{2}{*}{$\frac{\text { Events }}{7}$} & \multirow{2}{*}{$\frac{\text { Total }}{24}$} & & & & & \\
\hline & & & & & & $2.51[1.29,4.88]$ & & $\longrightarrow$ & \\
\hline Chen XJ 2012 & 22 & 33 & 10 & 32 & $6.4 \%$ & $2.13[1.21,3.76]$ & & 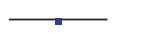 & \\
\hline Chen YL 2007 & 29 & 40 & 18 & 40 & $11.4 \%$ & $1.61[1.09,2.38]$ & & $\longrightarrow$ & \\
\hline Gao YH 2011 & 21 & 30 & 10 & 20 & $7.6 \%$ & $1.40[0.85,2.30]$ & & - & \\
\hline Hu JY 2015 & 26 & 30 & 12 & 30 & $7.6 \%$ & $2.17[1.37,3.43]$ & & & \\
\hline Li WS 2009 & 18 & 23 & 3 & 23 & $1.9 \%$ & $6.00[2.04,17.61]$ & & & \\
\hline Lu J 2007 & 27 & 54 & 6 & 30 & $4.9 \%$ & $2.50[1.16,5.37]$ & & & \\
\hline Ning F 2013 & 39 & 60 & 28 & 60 & $17.7 \%$ & $1.39[1.00,1.93]$ & & & \\
\hline Wang WM 2014 & 26 & 30 & 14 & 30 & $8.9 \%$ & $1.86[1.24,2.79]$ & & & \\
\hline Wu JM 2004 & 41 & 45 & 23 & 45 & $14.6 \%$ & $1.78[1.32,2.41]$ & & & \\
\hline Wu L 2005 & 23 & 32 & 4 & 24 & $2.9 \%$ & $4.31[1.72,10.83]$ & & & \\
\hline Zhao WX 2001 & 29 & 42 & 17 & 36 & $11.6 \%$ & $1.46[0.98,2.18]$ & & & \\
\hline Total $(95 \%$ CI $)$ & & 445 & & 394 & $100.0 \%$ & $1.91[1.66,2.19]$ & & & \\
\hline Total events & 320 & & 152 & & & & & & \\
\hline Heterogeneity: $\mathrm{Chi}^{2}$ & $.58, \mathrm{df}=$ & $1(\mathrm{P}=\mathrm{C}$ & 12); $I^{2}=$ & & & 0.05 & 0.2 & 5 & 20 \\
\hline Test for overall effec & $9.25(\mathrm{P}$ & 0.0000 & & & & & neostigmine & acupuncture & \\
\hline
\end{tabular}

FIgURE 3: Comparison of the cure rate between acupuncture and neostigmine in the treatment of PUR.

cure rate than intramuscular injection of neostigmine in the treatment of postpartum urinary retention. The results of meta-analysis are shown in Figure 3.

3.4.2. Postvoiding Residual (PVR). Only two articles reported PVR [15, 21], one of which offered means and deviations [21]: the PVR after treatment was $35.45 \pm 13.25 \mathrm{ml}$ in the acupuncture group and $70.67 \pm 23.58 \mathrm{ml}$ in the neostigmine group (mean: -35.22 ; 95\% CI: $-44.90,-25.54$ ), which was even lower in the acupuncture group. In the other article [11], means and interquartile ranges were offered: the median and the interquartile range of PVR after treatment were $0 \mathrm{ml}$ and $1.25 \mathrm{ml}$, respectively, in the acupuncture group and $89.5 \mathrm{ml}$ and $241.25 \mathrm{ml}$ in the neostigmine group $(P<0.001)$, suggesting acupuncture had a better effectiveness.

3.4.3. Overall Effectiveness Rate. Overall effectiveness rate was reported in all the included 15 articles. The criterion of "effective" differed among different studies, which might have included ability to urinate after treatment but with the presence of discomfort or a PVR of 50-300 $\mathrm{ml}$. Therefore, the heterogeneity among these studies was large. Test for between-studies heterogeneity of 15 studies yielded an $I^{2}$ of $70 \%$. The pooled cure rate was $93.24 \%$ in the acupuncture group and $72.75 \%$ in the neostigmine group; the RR (and 95\% CI) was $1.28(1.16,1.42)$ when these two groups were compared in the random-effect model, suggesting that acupuncture had a higher effectiveness rate than intramuscular injection of neostigmine in the treatment of postpartum urinary retention. The results of meta-analysis are shown in Figure 4.

3.4.4. Adverse Events. Seven of the 15 studies reported adverse events. Among them 6 studies [11, 12, 14, 19, 21, 24] reported that there was no adverse event. In the remaining one study, one patient from the neostigmine group suffered from full-body tremor 5 minutes after the intramuscular injection of neostigmine, which was spontaneously resolved
10 minutes later; no other abnormality was found except for slightly higher blood pressure and heart rate; in contrast, no adverse event was found in the acupuncture group [15].

3.5. Publication Bias. Over ten articles described pooled cure rate and effectiveness rate, and their publication biases were analyzed by using funnel plot analysis. The inverted funnel in Figure 5 is asymmetrical between left and right, suggesting that there may be a publication bias.

\section{Discussion}

4.1. Principal Findings. Based on our meta-analysis, the cure rate and effectiveness rate were significantly better in the acupuncture alone group than in intramuscular injection of neostigmine alone group (RR and its 95\% CI: 1.91 [1.66, $2.19]$ and $1.28[1.16,1.42]$, respectively). Thus, although intramuscular injection of neostigmine is the most commonly used drug for postpartum urinary retention, its efficacy is suboptimal; instead, acupuncture can achieve better curative effect. Moreover, no adverse event was reported in the acupuncture group, demonstrating acupuncture is safe and reliable. Therefore, acupuncture is more effective and safe in treating postpartum urinary retention.

4.2. The Limitations of These Studies. (1) This analysis only included clinical studies comparing acupuncture alone versus intramuscular injection of neostigmine. Studies on other nondrug traditional Chinese therapies (moxibustion therapy) or combined therapies were not included, which was based on the following two considerations. First, moxibustion can produce a bitter taste, which may have certain effects on the newborns who typically are staying with the mothers in the same ward. Second, the combined therapies are more complex than monotherapies. If acupuncture alone can achieve good effectiveness without much cost and time, no other therapy is necessary. (2) The disease severity, definition 


\begin{tabular}{|c|c|c|c|c|c|c|c|c|}
\hline \multirow{3}{*}{$\begin{array}{l}\text { Study or Subgroup } \\
\text { Bao FF } 2010\end{array}$} & \multicolumn{2}{|c|}{ Experimental } & \multicolumn{2}{|c|}{ Control } & \multirow{2}{*}{ Weight } & \multirow{2}{*}{$\begin{array}{c}\text { Risk Ratio } \\
\text { M-H, Random, 95\% CI }\end{array}$} & \multirow{2}{*}{\multicolumn{2}{|c|}{$\begin{array}{c}\text { Risk Ratio } \\
\text { M-H, Random, 95\% CI }\end{array}$}} \\
\hline & Events & Total & Events & Total & & & & \\
\hline & 24 & 26 & 17 & 24 & $6.0 \%$ & $1.30[0.99,1.72]$ & & \\
\hline Chen XJ 2012 & 23 & 32 & 21 & 32 & $5.1 \%$ & $1.10[0.79,1.53]$ & & \\
\hline Chen YL 2007 & 39 & 40 & 35 & 40 & $9.4 \%$ & $1.11[0.98,1.27]$ & & - \\
\hline Gao YH 2011 & 30 & 30 & 17 & 20 & $7.8 \%$ & $1.18[0.97,1.44]$ & & \\
\hline Hu JY 2015 & 29 & 30 & 15 & 30 & $4.6 \%$ & $1.93[1.34,2.78]$ & & \\
\hline Kang N 2012 & 19 & 20 & 10 & 20 & $3.5 \%$ & $1.90[1.21,2.98]$ & & \\
\hline Li WS 2009 & 22 & 23 & 11 & 23 & $3.6 \%$ & $2.00[1.29,3.09]$ & & \\
\hline Lu J 2007 & 49 & 54 & 22 & 30 & $7.0 \%$ & $1.24[0.98,1.56]$ & & \\
\hline Ning F 2013 & 57 & 60 & 51 & 60 & $9.5 \%$ & $1.12[0.99,1.26]$ & & 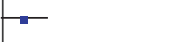 \\
\hline Teng HY & 32 & 34 & 21 & 32 & $6.3 \%$ & $1.43[1.10,1.87]$ & & \\
\hline Wang WM 2014 & 29 & 30 & 21 & 30 & $6.7 \%$ & $1.38[1.08,1.76]$ & & \\
\hline Wu JM 2004 & 42 & 45 & 36 & 45 & $8.5 \%$ & $1.17[0.99,1.38]$ & & \\
\hline Wu L 2005 & 30 & 32 & 12 & 24 & $3.9 \%$ & $1.88[1.24,2.83]$ & & \\
\hline You H 2003 & 46 & 48 & 19 & 20 & $9.6 \%$ & $1.01[0.90,1.13]$ & & \\
\hline Zhao WX 2001 & 41 & 42 & 29 & 36 & $8.5 \%$ & $1.21[1.03,1.43]$ & & \\
\hline Total (95\% CI) & & 546 & & 466 & $100.0 \%$ & $1.28[1.16,1.42]$ & & \\
\hline Total events & 512 & & 337 & & & & & \\
\hline \multicolumn{7}{|c|}{$\begin{array}{l}\text { Heterogeneity: } \mathrm{Tau}^{2}=0.02 ; \mathrm{Chi}^{2}=46.75, \mathrm{df}=14(\mathrm{P}<0.0001) ; \mathrm{I}^{2}=70 \% \\
\text { Test for overall effect: } \mathrm{Z}=4.75(\mathrm{P}<0.00001)\end{array}$} & $\begin{array}{c}0.5 \quad 0.7 \\
\text { neostigmine }\end{array}$ & $\begin{array}{lcc}1 & 1.5 \quad 2 \\
\text { acupuncture }\end{array}$ \\
\hline
\end{tabular}

FIGURE 4: Comparison of the effectiveness rate between acupuncture and neostigmine in the treatment of PUR.

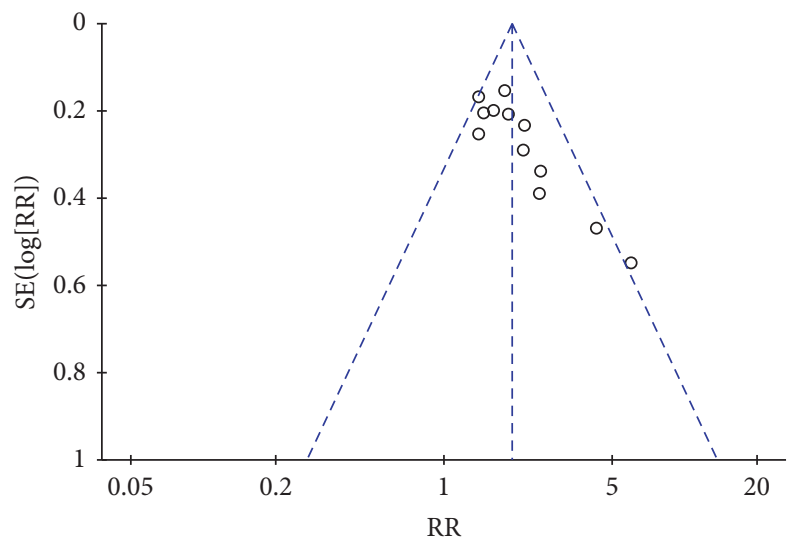

FIGURE 5: Funnel plot analysis of the cure rate.

of "effectiveness rate," and dosage of intramuscular injection of neostigmine differed among different studies. Therefore, there are certain heterogeneities among these studies. The objective of our current analysis was to evaluate the efficacy of acupuncture with the most commonly used drug. If the acupoints and dosage of drugs must be fully consistent, it would be impossible to determine whether most acupuncture studies are valid or not. (3) In some of the studies included, the specific randomization method was not described, blinding was not adopted, and the specific values of PVR were not reported; therefore, the methodological quality of these studies was average. (4) There was no study that compared acupuncture with blank control or placebo, so we did not know the effect of natural cause of the disease and the efficacy of acupuncture.

4.3. Implications for Clinical Practice and Further Research. The results of these studies have demonstrated that, as a safe and simple therapy, acupuncture alone has a better efficacy than intramuscular injection of neostigmine. The main acupoints used in these studies included sanyinjiao (SP6), zhongji (CV3), zusanli (S36), guanyuan (CV4), yinlingquan (SP9), sanjiao (CO17), shuidao (S28), and zhiyin (B67). Since TCM departments have been established in most maternity hospitals and general hospitals in China, it is easy and feasible to apply this treatment.

The included studies were limited by their small sample sizes and relatively low methodological quality. Therefore, prospective randomized controlled trials with larger scale and more rigorous design should be carried out to further validate the clinical efficacy of acupuncture in the treatment of postpartum urinary retention. Future studies should also be focused on the screening of fixed and effective acupoints, so as to benefit more mothers with this condition.

\section{Conclusion}

This review of 15 randomized trials shows that acupuncture is more effective and safe in treating postpartum urinary retention compared with intramuscular injection of neostigmine. However, the beneficial findings are inconclusive due to generally moderate evidence, and further large, rigorous trials are still warranted.

\section{Conflicts of Interest}

The authors have declared no conflicts of interest.

\section{Authors' Contributions}

Xiao-Mei Wang and Jing Gong contributed equally to this work. 


\section{Acknowledgments}

The authors thank Jing Li and Yu-Yan Pan from Beijing University of Chinese Medicine for their data extraction work for this article. This work is supported by Beijing University of Chinese Medicine (2016-DZM111-JC011).

\section{References}

[1] Nanyang Traditional Chinese Medicine School and Chongqing Traditional Chinese Medicine School, Obstetrics and Gynecology in Integrated Chinese and Western Medicine, vol. 227, Press of Traditional Chinese Medicine, Beijing, China, 2001.

[2] F. E. M. Mulder, K. O. Rengerink, J. A. M. van der Post, R. A. Hakvoort, and J.-P. W. R. Roovers, "Delivery-related risk factors for covert postpartum urinary retention after vaginal delivery," International Urogynecology Journal, vol. 27, no. 1, pp. 55-60, 2016.

[3] S.-K. Yip, G. Brieger, L.-Y. Hin, and T. Chung, "Urinary retention in the post-partum period. The relationship between obstetric factors and the post-partum post-void residual bladder volume," Acta Obstetricia et Gynecologica Scandinavica, vol. 76, no. 7, pp. 667-672, 1997.

[4] K. L. Musselwhite, P. Faris, K. Moore, D. Berci, and K. M. King, "Use of epidural anesthesia and the risk of acute postpartum urinary retention," American Journal of Obstetrics \& Gynecology, vol. 196, no. 5, pp. 472-475, 2007.

[5] P. Hee, G. Lose, R. Beier-Holgersen, E. Engdahl, and P. Falkenløve, "Postpartum voiding in the primiparous after vaginal delivery," International Urogynecology Journal, vol. 3, no. 2, pp. 95-99, 1992.

[6] Z. C. Huang, Y. Jing, X. M. Chen, and D. N. Chen, "Etiological analysis and prevention of postpartum urinary Retention," Contemporary Medicine, vol. 17, no. 26, pp. 54-55, 2011.

[7] G. Y. Cao, B. He, F. P. Wang et al., "Six Sigma management in lowering the incidence of nosocomial infection after indwelling catheterization," Journal of Nurse Training, vol. 29, no. 4, pp. 330-332, 2014.

[8] Y. S. Li, "Treatment of postpartum urinary retention with acupuncture: report of 44 cases," China Health Industry, vol. 9, no. 30, article 171, 2012.

[9] Y. J. Zhang, "Advances in acupuncture treatment of postpartum urinary retention," Shanghai Journal of Acupuncture and Moxibustion, vol. 30, no. 07, pp. 504-507, 2011.

[10] J. P. T. Higgins and S. Green, Eds., Cochrane Handbook for Systematic Reviews of Interventions, The Cochrane Collaboration and John Wiley \& Sons Ltd., 2008.

[11] F. F. Bao and A. J. Yuan, "Treatment of postpartum urinary retention with acupuncture in 26 cases," Jiangxi Journal of Traditional Chinese Medicine, vol. 41, no. 08, article 55, 2010.

[12] X. J. Chen and W. Zheng, "Treatment of 33 cases of postpartum urinary retention with electroacupuncture sparse-dense wave," Guangming Journal of Chinese Medicine, vol. 27, no. 01, pp. 9293, 2012.

[13] Y. L. Chen, "Electroacupuncture of postpartum urinary retention in 40 cases," Shanghai Journal of Acupuncture and Moxibustion, vol. 1, article 33, 2007.

[14] Y. H. Gao, "Therapeutic effect of electroacupuncture on postpartum urinary retention: report of 30 cases," Inner Mongolia Journal of Traditional Chinese Medicine, vol. 30, no. 11, article 80, 2011.
[15] J. Y. Hu, "Clinical observation on treatment of postpartum urinary retention by acupuncture," Zhejiang University of Traditional Chinese Medicine, 2015.

[16] N. Kang, "Clinical observation on treatment of postpartum urinary retention by electroacupuncture on acupoints," Chinese Journal of Chinese Medicine, vol. 27, no. 02, pp. 250-251, 2012.

[17] W. S. Li, "Treatment of refractory postpartum urinary retention with acupuncture," Chinese and Foreign Health Abstracts, vol. 6, no. 24, pp. 279-279, 2009.

[18] J. Lu, "Clinical observation on the treatment of 54 cases of postpartum urinary retention by acupuncture on foot Jue Yin meridian," Journal of Shanxi College of Traditional Chinese Medicine, no. 05, pp. 41-42, 2007.

[19] F. Ning and H. S. Zhang, "linical observation on the treatment of postpartum urinary retention by acupuncture with "Visceratime Modulation Method"," Chinese Medicine Modern Distance Education of China, vol. 11, no. 31, pp. 51-52, 2013.

[20] H. Y. Teng, "Clinical observation on the treatment of postpartum urinary retention by electroacupuncture," Medicine Frontier, vol. 7, no. 30, pp. 65-66, 2017.

[21] W. M. Wang, "Clinical observation of 30 cases of postpartum urinary retention treated by abdominal acupuncture combined with scalp acupuncture," Massage and Rehabilitation Medicine, vol. 3, pp. 88-89, 2014.

[22] J. M. Wu, "Treatment of 90 cases of postpartum urinary retention with acupuncture on acupoints," Journal of Clinical Acupuncture and Moxibustion, vol. 20, no. 3, article 24, 2004.

[23] L. Wu, "Clinical observation on treatment of postpartum urinary retention by electroacupuncture," Journal of External Therapy of Traditional Chinese Medicine, vol. 14, no. 3, article 46, 2005.

[24] H. You, X. B. He, and J. M. Luo, "Clinical observation of 68 cases with postpartum urinary retention treated with body acupuncture and auricular acupuncture," Hubei Journal of Traditional Chinese Medicine, vol. 25, no. 4, pp. 44-45, 2003.

[25] Y. Zhao and S. F. Yue, "Clinical observation on treating postpartum urinary retention with scalp acupuncture as a main treatment," Guangming Journal of Chinese Medicine, vol. 16, no. 4, pp. 36-37, 2001. 


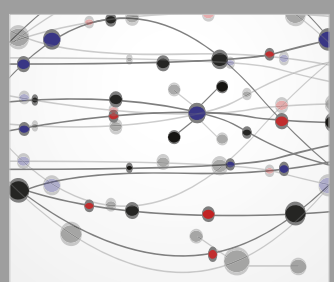

The Scientific World Journal
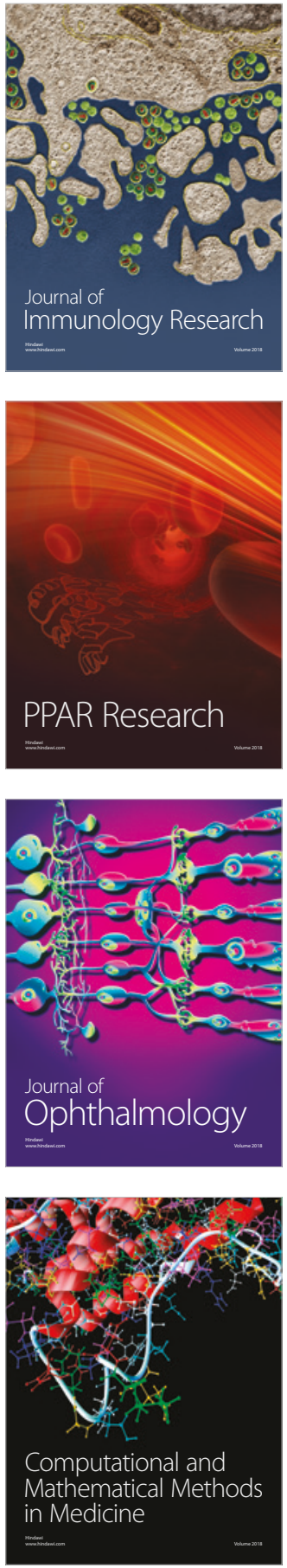

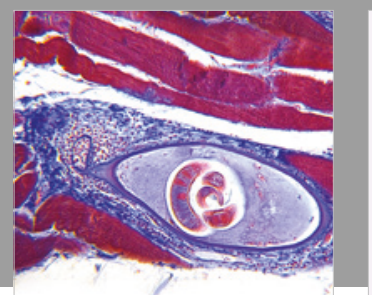

Gastroenterology Research and Practice

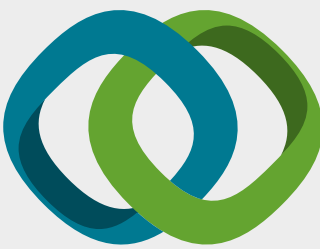

\section{Hindawi}

Submit your manuscripts at

www.hindawi.com
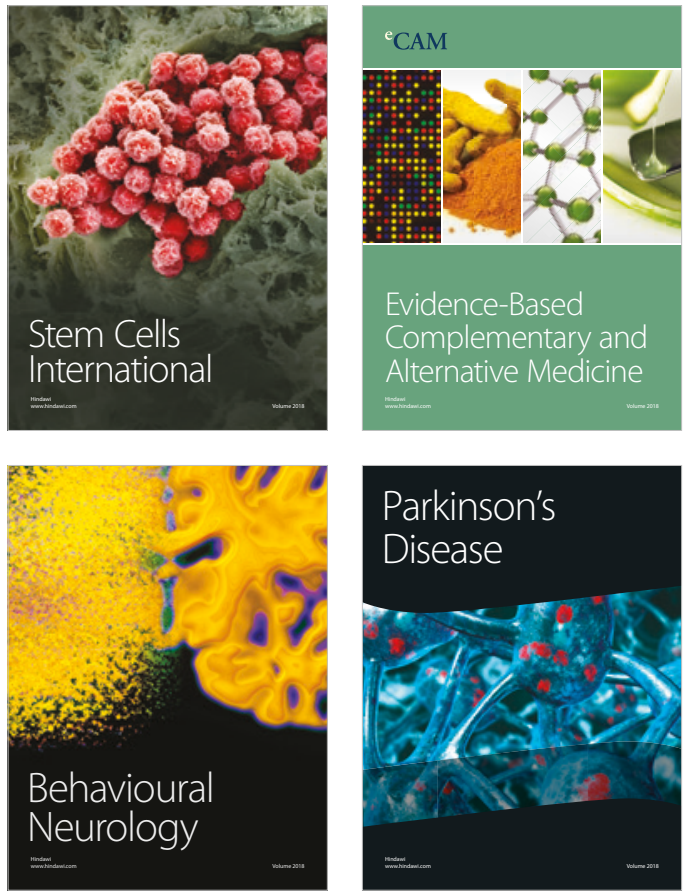

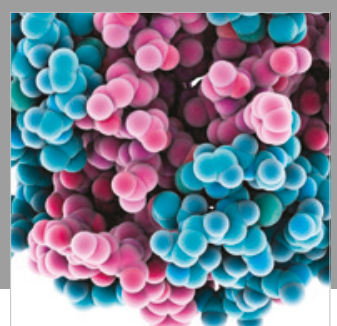

ournal of

Diabetes Research

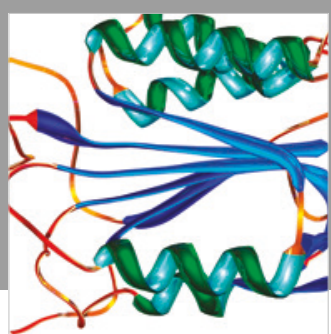

Disease Markers
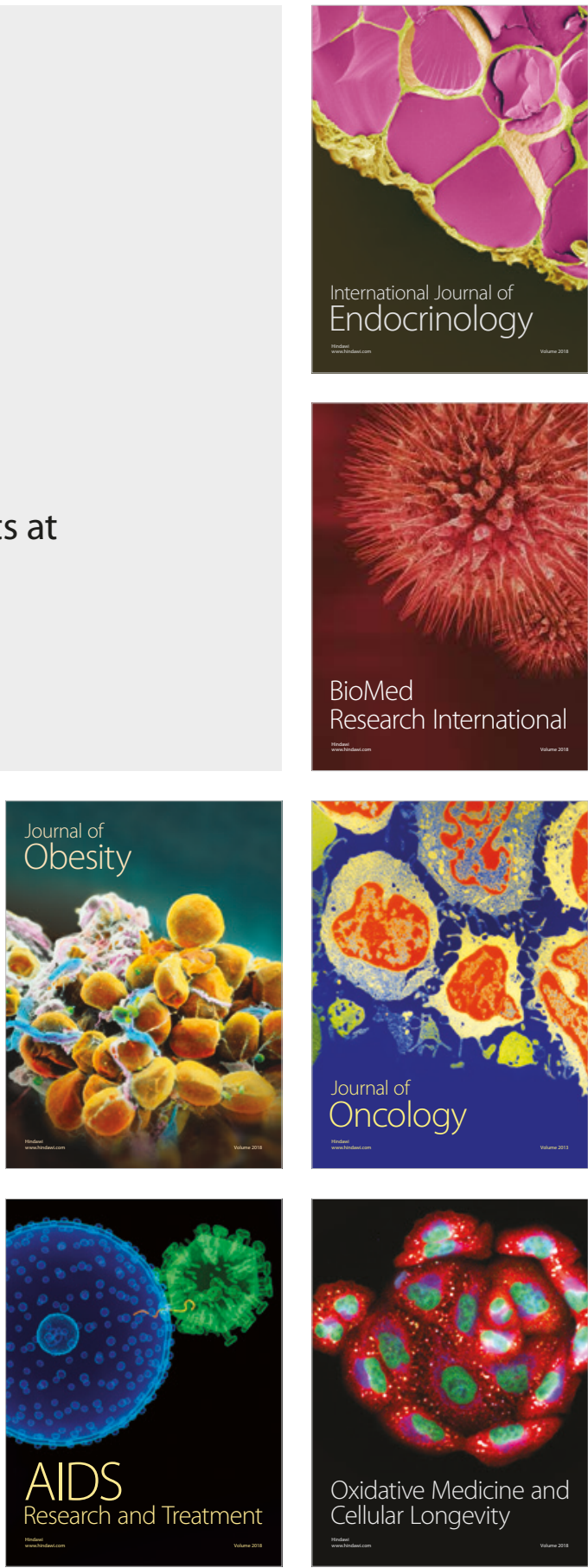